\title{
An innovative modification of the nutrient medium formulation for the isolation and differentiation of enterobacteria
}

\author{
Vladimir Ermakov and Nikolay Titov \\ Samara State Agrarian University, 2, Uchebnaya str., Ust-Kinelsky, Kinel, 446442, Russia
}

\begin{abstract}
In laboratory conditions, the modified nutrient differential diagnostic medium Drigalski agar with lactose is recommended to be used for cultivation (isolation) and differentiation of enterobacteria of the Enterobacteriaceae family. The differentiation of enterobacteria on a modified medium is carried out according to their ability to ferment lactose, mannitol, glucose, sucrose, gelatin and form hydrogen sulfide. The environment can also be used for conducting sanitary and microbiological studies of environmental objects. The environment can be used to perform the ONPG test. In the course of preparing the modified Drigalski agar for operation in laboratory conditions, we recommend using the medium according to one of the options: when one of the carbohydrates is added, the medium is poured into single-section reusable or disposable petri dishes; when two carbohydrates are added, the medium is poured into two-section reusable or disposable petri dishes; when a complex of carbohydrates lactose + mannitol and glucose + sucrose is added, the medium is poured into two-section reusable or disposable petri dishes; when using all four drives separately, the medium is dispensed into single-compartment reusable or disposable petri dishes. We recommend preparing modified Drigalski agar at a plant for the production of ready-made culture media with filling the medium in two-section petri dishes (complex of carbohydrates lactose + mannitol in one section, glucose + sucrose in another section), or with filling the medium in four-section petri dishes (with each carbohydrate in separate section).
\end{abstract}

\section{Introduction}

Today, improving the means for assessing the indicators of animal microbiocenosis, diagnostics, prevention, treatment of non-communicable and infectious diseases is the most significant task facing veterinary specialists, microbiologists and biotechnologists [1,2].

According to the WHO, every year in the world, more than 2 billion people fall ill with acute intestinal infections. The etiological structure of AEI in different countries differs significantly, and not rarely, despite the modern capabilities of laboratory microbiological means and methods, is not established [3, 4].

Pathogenic Salmonella, Escherichia, Shigella, Campylobacter and Helicobacterium have long been known to microbiologists, virologists, infectious disease specialists and therapists. However, in the infectious pathology of humans and animals, their role has undergone certain changes. At the same time, the incidence of such infections has increased and the clinical spectrum of their manifestation has expanded. According to leading microbiologists and infectious disease specialists, this was facilitated by a certain progress in the field of laboratory and clinical diagnostics. On the other hand, this was caused by the anthropogenic transformation of the external environment, which directly affects the conditions of reproduction and the routes of infection transmission, as

\footnotetext{
* Corresponding author: Vladimir_21_2010@mail.ru
}

well as the susceptibility of various risk groups $[5,6,7$, $8,9]$.

At the same time, at the very beginning of the $21 \mathrm{st}$ century, a significant update and replenishment took place in the Enterobacteriaceae family. For example, in 2001 , based on the analysis of the $16 \mathrm{~S}$ rRNA and rpoB gene sequences, the bacteria $\mathrm{K}$. planticula and $\mathrm{K}$. terrigena were isolated into the new genus Raoultella while maintaining their species designation Raoultella planticula spp. nov. and Raoultella terrigena spp. nov. Further, in 2004, during the sequencing of genomes and analysis of the nucleotide sequences of the genes $16 \mathrm{~S}$ rRNA, rpoB, gyrA, mdh, infB, phoE, and nifH, five new species K. ornithinolytica, K. variicola, K. singaporensis, $\mathrm{K}$. milletis and $\mathrm{K}$. senegalensis were added to the Klebsiella genus that are often found in the environment. Subsequently, several new groups (genera) of enterobacteria were introduced into the Enterobacteriaceae family. These are the bacteria Cedecea, Ewingella, Kluyvera, Lecrecia, Moellerella, Pantoea, Pragia, Rahnella, Tatumella, Xenorhabdus and Jokenella. The clinical significance of these new members of the Enterobacteriaceae family is under investigation. The biological properties of these enterobacteria are studied, methods of their isolation and identification are being developed [5, 6].

Diagnosis of infectious diseases is one of the most difficult problems in clinical medicine. Laboratory research methods in a number of nosological forms play a leading and, in a number of clinical situations, a 
decisive role not only in terms of diagnosis, but also in determining the final outcome of the disease. The diagnosis of infectious diseases almost always involves the use of a set of laboratory methods. Moreover, the bacteriological group of methods is one of the three leading in the diagnosis of infectious diseases. One of the important elements in the laboratory diagnosis of AEIs and opportunistic infections caused by enterobacteria is the isolation of the pathogen in a pure culture on nutrient media. Culture media are a basic element in laboratory diagnosis of infectious pathologies. The media should be formulated to optimally ensure the growth and reproduction of microorganisms of a particular species or family. The intensive development of biotechnology and microbiology makes it possible today to develop new nutrient media and modify existing media formulations $[10,11,12,13,14]$.

In the course of creating new and modifying old formulations of nutrient media, it is also necessary to take into account the growing anthropogenic impact on the environment, which leads to an increase in the frequency of mutations in microorganisms with the appearance of new properties in them. In this regard, the design and production of high-quality nutrient media, the development of formulations for new microbiological media and the improvement of already used media are one of the important areas of work in the field of biotechnology, medical and veterinary microbiology $[15$, $16,17]$.

\section{Materials and methods}

Research stages. During the first stage, the isolation, cultivation and identification of enterobacteria cultures obtained from various animal species were carried out and the sensitivity of enterobacteria to antimicrobial drugs was revealed. The factors of pathogenicity and persistence in the identified cultures of enterobacteria were studied. In the course of the second stage, the formulations of nutrient microbiological media for enterobacteria, enrichment media and differential diagnostic media were analyzed. The nutrient base and shaper, growth stimulants, carbohydrates, an indicator, selective components and growth inhibitors of accompanying microflora for the modification of Drigalski Lactose Agar with lactose (Drigalski Lactose Agar, AppliChem A5731.0500) were selected.

The material for research was 253 isolates of enterobacteria, isolated from the intestinal microbiotope of various farm animals (cows, sheep, goats, pigs, horses, chickens and geese), wild animals (wild boars, moose, foxes), zoo animals (pony, camel), pets (cats, dogs, ferrets, chinchillas). The object for research was a modified by us differential diagnostic commercial nutrient medium Drigalski agar with lactose with a selective additive. As a basis for the modification, the recipe of the nutrient medium Drigalski Lactose Agar with lactose (Drigalski Lactose Agar, AppliChem A5731.0500) was taken, which is a differential diagnostic medium with selective properties intended for the isolation and differentiation of bacteria of the Enterobacteriaceae family and some non-fermenting microorganisms in the test samples.

Isolation, cultivation and identification of enterobacteria cultures were carried out on specific nutrient media, including on Drigalski Lactose Agar (AppliChem), and on Drigalski Lactose Agar with our modification. Pure cultures of enterobacteria were identified by morphological, tinctorial, cultural, biochemical, and serological properties. Biochemical identification of enterobacteria was carried out using the API identification method, microtest system produced by NPO Nutrient Media and NPO Diagnostic Systems: PBDE plates (plate for biochemical differentiation of enterobacteria), microtest system for biochemical identification of enterobacteria (LTC-M-12E), microtest system for determining the enzymatic activity of enterobacteria (MTS-5U), microtest system for biochemical identification of Shigella Sonnei biovars (MTS-Sonnei), microtest system for biochemical identification of Salmonella (MTS-Salm), microtest system for biochemical identification of Shigella Newcastle (MTS-Newcastl). The API identification system (bioMerieux, France) is based on biochemical tests and is used as a reference identification method in the world. API systems are the closest to the classical biochemical series, but at the same time they have a number of indisputable advantages over them. To identify enterobacteria, we used API $20 \mathrm{E}$ strips to identify members of the Enterobacteriaceae family and other Gram-negative bacilli (the most clinically significant non-fermenting microorganisms), API $10 \mathrm{~S}$ to identify members of the Enterobacteriaceae family and other Gram-negative bacilli (shortened strip, consisting of 10 tests) and RapiD strip $20 \mathrm{E}$ to identify representatives of the Enterobacteriaceae genus. The persistence factors of enterobacteria was determined by conventional methods. The research results were statistically processed according to the generally accepted method using a Pentium PC using the Microsoft Office Excel application.

\section{Results and discussion}

During the research, 130 intestinal isolates of enterobacteria were isolated from farm animals (cows, sheep, goats, pigs, horses, chickens and geese), 35 intestinal isolates of enterobacteria from wild animals (wild boars, moose, foxes), 23 intestinal isolates of enterobacteria from zoo animals (pony, camel), 65 intestinal isolates of enterobacteria from pets (cats, dogs, ferrets, chinchillas).

In the species composition of intestinal isolates of enterobacteria, Escherichia coli, Shigella dysenteriae, Shigella flexneri, Salmonella enteritidis, Klebsiella oxytoca, Proteus vulgaris, Providencia alcalifacien, Hafnia alvei, Morganella morganii, Enterobacter cloaciaae, Yersinia enterocolitica were identified.

The species composition of enterobacteria isolated from farm animals was dominated by representatives of the genus Escherichia coli $46.83 \%$ and Serratia 
marcescens $30.62 \%$. In the species composition of enterobacteria isolated from wild animals, representatives of the genus Escherichia coli $(41.28 \%)$ and Serratia marcescens $(35.14 \%)$ dominated. The species composition of enterobacteria isolated from zoo animals was dominated by representatives of the genus Escherichia coli $33.03 \%$ and Serratia marcescens $42.48 \%$. In the species composition of enterobacteria isolated from pets, representatives of the genus Escherichia coli (43.94\%) and Serratia marcescens (33.87\%) dominated.

In the process of identifying persistence factors in enterobacteria, isolated from various animals, the indicators of manifestation of antilysozyme, anticarnosine activity and the ability of enterobacteria to biofilm formation were determined. Enterobacteria possessing persistence factors can survive (survive) in the macroorganism of humans and animals for an unlimited time, protecting themselves from cellular and humoral factors of nonspecific protection of the macroorganism. At the same time, the presence of persistence factors in enterobacteria allows them to exhibit pathogenic properties.

Enterobacteria of the genus Klebsiella oxytoca and Salmonella enteritidis exhibited the highest antilysozyme and anticarnosine activity compared to other members of the Enterobacteriaceae family. Escherichia coli and Klebsiella oxytoca were most capable of biofilm formation.

The lowest values of antilysozyme activity were recorded in Enterobacter cloacae, and anticarnosine activity was lowest in Morganella morganii. At the same time, the pathogenic Salmonella enteritidis showed the lowest ability to form biofilm in comparison with other enterobacteria.

Pathogenic Yersinia enterocolitica, Shigella dysenteriae and Shigella flexneri also exhibited relatively high antilozyme, anticarnosine activity and biofilm formation against the background of persistence indicators of other enterobacterial cultures. However, the ability of Yersinia enterocolitica to biofilm was lower than that of Proteus vulgaris.

Trimethoprim exhibited the greatest antimicrobial activity against all the enterobacteria cultures isolated by us. Most enterobacteria were resistant to metronidazole. At the same time, pathogenic Shigella dysenteriae, S. flexneri, Salmonella Enteritidis and Yersinia enterocolitica were also more sensitive to trimethoprim.

In the course of creating a new modification of lactose agar, the selection of the nutrient base and the shaper of the nutrient medium was carried out. In microbiological practice, agar-agar is most often used for shaping in the formulation of nutrient media. In the formulation of solid nutrient media, agar is introduced, as a rule, in a volume of $1-2 \%$, since it forms a gel in water well and gives the medium a stable shape after solidification. Agar melts at $80-100{ }^{\circ} \mathrm{C}$ and solidifies at $37-40{ }^{\circ} \mathrm{C}$. It is most advisable to use pure agar intended directly for microbiological purposes.

As an agar base for the modification of lactose agar, we used dry bacteriological agar produced by the FBSI SSC MPB Obolensk (Russia).
In the course of choosing a nutrient base and growth stimulants, the usefulness of the components in terms of a set of nutrients and their availability for microorganisms were taken into account. Meat water, beef extract, peptic digest of animal tissue, Hottinger's tryptic digest, pancreatic hydrolyzate of casein, acid hydrolyzate of casein, hydrolyzate of kerata mucus pancreatic, hydrolyzate of fishmeal, tryptone, feed or bread (baker's) yeast extract.

When choosing a protein hydrolyzate for the nutrient base of the medium, we took into account the method of obtaining and the degree of protein breakdown. For the modification of lactose agar, we used pancreatic hydrolyzate of casein and pancreatic hydrolyzate of fish meal produced by FBSI SSC MPB Obolensk (Russia).

The pancreatic hydrolyzate of casein, obtained by hydrolysis of casein with pancreatin, is somewhat inferior in amine nitrogen to the acid hydrolyzate of casein of an average degree of degradation (3.0-4.0\% versus $6.0-6.5 \%$, respectively). Amino nitrogen is the total amount of free amino acids in a protein hydrolyzate. The amino acid content of casein acid hydrolyzate is slightly higher, but tryptophan is absent. In pancreatic casein hydrolyzate, tryptophan $1.0-1.1 \%$ is present. However, it should be borne in mind that acid hydrolysis denatures some useful components, for example, carbohydrates, tryptophan, cystine and other substances. At the end of acid hydrolysis, the concentration of salts increases. In this regard, pancreatic hydrolysis using pancreatin is more nutrient-friendly and provides sufficient protein breakdown for enterobacteria growth and reproduction.

Pancreatic hydrolyzate of fishmeal in the content of peptides $(67.6 \%)$ is inferior to pancreatic hydrolyzate of gelatin $(103.7 \%)$ and blood $(71.0 \%)$, but surpasses pancreatic hydrolysates of soy, soy concentrate and gluten. In terms of amine nitrogen content, pancreatic hydrolyzate of fishmeal is second only to pancreatic hydrolyzate of gelatin and blood, surpassing pancreatic hydrolyzate of soy, soy concentrate and gluten in this indicator.

An important factor is also the fact that pancreatic hydrolyzate of casein, acid hydrolyzate of casein of medium degree of degradation, hydrolyzate of pancreatic sprat and fish meal are produced by various factories in Russia directly for microbiological purposes.

In order to enrich the nutrient base with high molecular weight peptides, enzymatic bacteriological peptone with a high tryptophan content produced by HiMedia Laboratories (India) was introduced into the medium formulation. Peptone obtained by enzymatic hydrolysis using pepsin and trypsin contains a large amount of high molecular weight peptides and amino acids.

It was decided to introduce an aminopeptide, an extract of bread yeast, as stimulants of the growth of enterobacteria in the formulation of the nutrient medium.

The microbiological aminopeptide was introduced into the medium formulation as a nitrogen source containing a full set of amino acids and low molecular weight peptides. Peptides and amino acids are not only the main source of nitrogen, but also provide 
enterobacteria with phosphorus, sulfur, various vitamins and other growth substances required by enterobacteria for active growth and reproduction.

The aminopeptide replaces the meat extract found in Drigalski Lactose Agar. Aminopeptide is more nutritious in the presence of amine nitrogen, peptides, amino acids and other growth factors in comparison with meat water, mesopatamia broth, meat extract and Hottinger tryptic digest. Amino nitrogen is the total amount of free amino acids in a protein hydrolyzate. At the same time, the meat extract and meat water contain various carbohydrates, which can adversely affect the results of enterobacteria differentiation based on the fermentation of carbohydrates.

Bread (baker's) yeast extract, produced by NPO Nutrient Media (Makhachkala), enriches the environment with water-soluble B vitamins, free amino acids, purine and pyrimidine bases. At the same time, the extract of bread yeast is superior in vitamin value to the extract of feed yeast.

As a result of the analysis of the biological usefulness of various nutritional bases and growth factors, we decided to include the following in the modified formulation of lactose agar: bacteriological agar, pancreatic hydrolyzate of fishmeal, pancreatic hydrolyzate of casein, enzymatic bacteriological peptone with a high content of tryptophan, aminopeptide and bread yeast extract.

In the course of the choice of mineral components, the need for enterobacteria, their interaction with other components of the environment, the expected diagnostic informativeness and inhibitory effect on the accompanying microflora were taken into account. Sodium chloride is necessary to create isoosmotic conditions in the environment that are optimal for the growth and reproduction of microorganisms. Sodium chloride inhibits the growth of concomitant grampositive streptococci.

Sodium carbonate (soda ash), readily soluble in water to create an alkaline medium, is used to adjust the $\mathrm{pH}$ of the nutrient medium.

Sodium sulfite (sodium sulfite, preservative E 221) is a good reducing agent and is highly soluble in water. Sodium sulfite enters into a chemical reaction with substances formed during the fermentation of lactose by lactose-positive enterobacteria, which leads to the color of the colonies and serves as a differentiating feature.

Sodium thiosulfate and iron (II) sulfate are necessary for the detection of hydrogen sulfide production in enterobacteria. Sodium thiosulfate (sodium sulfate, sodium thiosulfuric acid, sodium hyposulfite) is a derivative of thiosulfuric acid, is a white powder with crystalline structure, transparent crystals, salty-bitter taste, and is readily soluble in water.

Iron (II) sulfate is an inorganic compound, an iron salt of sulfuric acid, a colorless, opaque substance readily soluble in water.

Carbohydrates were introduced into the formulation of the medium for the differentiation of enterobacteria based on the fermentation of lactose, glucose, sucrose and mannitol. Carbohydrates lactose and mannitol are included in the recipe $\mathrm{A}$ of the medium, and glucose and sucrose are included in the recipe B of the medium. Carbohydrates are the best source of carbon for a wide range of microorganisms.

It was decided to introduce gelatin into the formulation of the medium in order to reveal the proteolytic activity in enterobacteria, in particular in representatives of the Proteus genus.

The dye fuchsin is essential for the differentiation of enterobacteria, which ferment mainly lactose.

As an indicator, the available and most frequently used in the formulations of differential diagnostic nutrient media, the Andrade indicator with the BP indicator were selected. The Andrade indicator contains sour fuchsin, sodium hydroxide $1 \mathrm{~N}$ and distilled water. The combined indicator BP consists of aqueous blue and rosolic acid (aurin, peonin or red coralline).

It was decided to use antibiotics in a selective supplement to the medium as selective inhibitors of the growth of the accompanying microflora.

The shape-former, nutrient bases, growth factors, carbohydrates, minerals chosen by us will fully meet the nutritional needs of enterobacteria.

Thus, the recipe, modified by us, lactose agar has a composition consisting of two recipes A and B (Table 1).

Working with our modified nutrient medium involves the use of two-section disposable Petri dishes. In this case, you can also use reusable glass Petri dishes with a partition that divides the dish into two equal sections. Component $\mathrm{A}$ of modified lactose agar is poured into one section, component $\mathrm{B}$ is poured into the other section.

In the course of the selection of selective components, we considered antibacterial drugs effective against concomitant gram-positive and gram-negative microflora. We selected vancomycin from the glycopeptide group, linezolid from the oxazolidinone group, and telithromycin from the ketolide group.

As a result, the minimum inhibitory concentration (MIC) of vancomycin and telithromycin for most enterobacterial cultures was $0.063 \mathrm{~g} / \mathrm{dm}^{3}$, and the MIC of linezolid was at the level of $0.031 \mathrm{~g} / \mathrm{dm}^{3}$. As a result, we have developed a selective additive to modified lactose agar includes vancomycin, linezolid, and telithromycin (Table 2). In this regard, it was decided to assign the following name to this additive "VLT selective additive". Dyes and indicators that are part of the culture medium can also inhibit the growth and reproduction of microorganisms. In this regard, it is necessary to select dyes and indicators in a concentration that does not have a bactericidal effect on microorganisms.

The dyes fuchsin basic, Congo red and water blue at a concentration of 0.05 to $50 \mathrm{~g} / \mathrm{dm}^{3}$ do not affect the growth and reproduction of the enterobacteria we isolated. At the same time, most of the tested dyes and indicators at a concentration of 1.0 to $50.0 \mathrm{~g} / \mathrm{dm}^{3}$ of the nutrient medium completely suppress or inhibit the growth and reproduction of enterobacteria.

In this regard, in the formulation of modified lactose agar, we included basic fuchsin as a dye, Andrade indicator and BP indicator. Considering that lactosepositive enterobacteria ferment lactose well, shifting, as a result of fermentation, the $\mathrm{pH}$ of the nutrient medium 
to the acidic side in the growth zone of the colony, further recovery of the main fuchsin is observed with the manifestation of pink or red coloration of lactose- positive colonies of microorganisms. Thus, there is a differentiation of lactose-positive and lactose-negative enterobacteria.

Table 1. Recipe of modified Drigalski Lactose Agar (AppliChem A5731,0500)

\begin{tabular}{|c|c|c|}
\hline Medium components & Recipe A $\left(\mathrm{g} / \mathrm{dm}^{3}\right)$ & Recipe B $\left(\mathrm{g} / \mathrm{dm}^{3}\right)$ \\
\hline Bacteriological agar & 12.0 & 12.0 \\
\hline Fishmeal Pancreatic Hydrolyzate & 5.0 & 5.0 \\
\hline Pancreatic Casein Hydrolyzate & 5.0 & 5.0 \\
\hline $\begin{array}{l}\text { Peptone enzymatic bacteriological with high tryptophan } \\
\text { content }\end{array}$ & 5.0 & 5.0 \\
\hline Aminopeptide & 2.0 & 2.0 \\
\hline Bread yeast extract & 2.0 & 2.0 \\
\hline Gelatin & 0.5 & 0.5 \\
\hline Sodium chloride & 5.0 & 5.0 \\
\hline Sodium carbonate & 0.5 & 0.5 \\
\hline Sodium sulfite & 0.5 & 0.5 \\
\hline Sodium thiosulfate & 0.3 & 0.3 \\
\hline Iron (II) sulfate & 1.0 & 10 \\
\hline Carbohydrate & Lactose 10.0 & Glucose 10.0 \\
\hline Carbohydrate & Mannitol 7.0 & Sucrose 7.0 \\
\hline Fuchsin basic & 1.0 & 1.0 \\
\hline Andrade indicator with BP indicator & 0.2 & 0.2 \\
\hline
\end{tabular}

Table 2. Composition of the VLT selective additive

\begin{tabular}{|c|c|}
\hline Antibacterial drugs & Concentration $\left(\mathrm{g} / \mathrm{dm}^{3}\right)$ \\
\hline Vancomycin & 0.008 \\
\hline Linezolid & 0.004 \\
\hline Telithromycin & 0.008 \\
\hline
\end{tabular}

Table 3. Time of cultivation of enterobacteria isolated from farm animals

\begin{tabular}{|l|l|c|c|}
\hline \multirow{2}{*}{ Enterobacteria cultures } & \multicolumn{3}{|c|}{ Cultivation time, $\mathrm{h}$} \\
\cline { 2 - 4 } & & $\begin{array}{c}\text { Drigalski Lactose Agar } \\
\text { (AppliChem) }\end{array}$ & $\begin{array}{c}\text { Modified Drigalski } \\
\text { Lactose Agar }\end{array}$ \\
\hline Escherichia coli & & & \\
\hline Salmonella Enteritidis & & $22.56 \pm 0.74$ & $20.34 \pm 0.85$ \\
\hline Klebsiella oxytoca & $22.82 \pm 1.12$ & $26.14 \pm 1.84$ & $20.76 \pm 1.12$ \\
\hline Proteus vulgaris & $28.34 \pm 3.26$ & $27.44 \pm 1.82$ & $22.28 \pm 0.94$ \\
\hline Providencia alcalifaciens & $26.54 \pm 2.32$ & $28.16 \pm 2.32$ & $21.52 \pm 1.35$ \\
\hline Hafnia alvei & $30.48 \pm 2.64$ & $29.18 \pm 2.66$ & $22.54 \pm 1.26$ \\
\hline Morganella morganii & $33.28 \pm 3.58$ & $27.36 \pm 2.52$ & $20.58 \pm 1.64$ \\
\hline Enterobacter cloacae & $28.56 \pm 2.74$ & $26.18 \pm 2.36$ & $23.66 \pm 0.78$ \\
\hline Citrobacter freundii & $28.66 \pm 2.52$ & $26.64 \pm 1.88$ & $22.68 \pm 1.32$ \\
\hline Serratia marcescens & $27.58 \pm 2.44$ & $27.12 \pm 2.52$ & $25.72 \pm 1.34$ \\
\hline Erwinia amylovora & $28.26 \pm 2.66$ & $27.48 \pm 2.38$ & $21.25 \pm 1.36$ \\
\hline Kluyvera cryocrescens & $25.74 \pm 2.78$ & $30.22 \pm 2.14$ & $18.28 \pm 1.56$ \\
\hline Yersinia enterocolitica & $32.58 \pm 3.42$ & $24.58 \pm 2.22$ & \\
\hline
\end{tabular}

The effectiveness of nutrient differential diagnostic media with a selective effect was revealed according to the time required for the formation of Enterobacteriaceae colonies of 1-3 $\mathrm{mm}$ in diameter on the media. The cultivation time of enterobacteria on commonly used nutrient media and on modified Drigalski agar with lactose was different. The cultivation time of enterobacteria isolated from farm animals ranged from $18.28 \pm 1.56$ to $33.28 \pm 3.58 \mathrm{~h}$ (Table 3 ).
The cultivation time of enterobacteria on our modified Drigalski agar with lactose ranged from $18.28 \pm 1.56 \mathrm{~h}$ for Yersinia enterocolitica to $25.72 \pm 1.34 \mathrm{~h}$ for Erwinia amylovora. The cultivation of enterobacteria on Drigalski Lactose Agar with lactose (Drigalski Lactose Agar, AppliChem) took from $20.36 \pm 1.78 \mathrm{~h}$ for Yersinia enterocolitica to $30.22 \pm 2.14 \mathrm{~h}$ for Erwinia amylovora. At the same time, almost all enterobacterial strains isolated from farm animals formed colonies on modified Drigalski agar with lactose within $24 \mathrm{~h}$, with 
the exception of Erwinia amylovora $(25.72 \pm 1.34 \mathrm{~h}) \mathrm{The}$ cultivation time of enterobacteria isolated from wild animals ranged from $16.28 \pm 1.44$ to $33.74 \pm 4.14 \mathrm{~h}$ (Table 4).

Table 4. Time of cultivation of enterobacteria isolated from wild animals

\begin{tabular}{|l|c|c|c|}
\hline \multicolumn{1}{|c|}{ Enterobacteria cultures } & \multicolumn{3}{|c|}{ Cultivation time, $\mathrm{h}$} \\
\cline { 2 - 4 } & Endo agar & $\begin{array}{c}\text { Drigalski Lactose Agar } \\
\text { (AppliChem) }\end{array}$ & $\begin{array}{c}\text { Modified Drigalski } \\
\text { Lactose Agar }\end{array}$ \\
\hline Escherichia coli & $21.32 \pm 0.75$ & $20.14 \pm 1.12$ & $16.28 \pm 1.44$ \\
\hline Shigella dysenteriae & $28.56 \pm 2.16$ & $25.32 \pm 1.74$ & $23.38 \pm 1.52$ \\
\hline Shigella flexneri & $33.42 \pm 1.72$ & $28.33 \pm 2.08$ & $26.76 \pm 1.88$ \\
\hline Salmonella Enteritidis & $25.56 \pm 1.18$ & $23.88 \pm 1.36$ & $20.44 \pm 1.06$ \\
\hline Klebsiella oxytoca & $27.38 \pm 1.84$ & $27.17 \pm 2.08$ & $22.86 \pm 1.12$ \\
\hline Proteus vulgaris & $28.44 \pm 1.57$ & $26.12 \pm 1.88$ & $18.59 \pm 2.04$ \\
\hline Providencia alcalifaciens & $28.87 \pm 2.18$ & $27.30 \pm 1.94$ & $20.34 \pm 0.94$ \\
\hline Hafnia alvei & $27.30 \pm 1.68$ & $25.22 \pm 1.60$ & $19.52 \pm 1.34$ \\
\hline Morganella morganii & $26.88 \pm 1.78$ & $24.56 \pm 1.80$ & $20.55 \pm 1.38$ \\
\hline Enterobacter cloacae & $29.18 \pm 1.33$ & $24.28 \pm 1.66$ & $20.83 \pm 1.90$ \\
\hline Citrobacter freundii & $26.08 \pm 1.82$ & $25.88 \pm 1.06$ & $24.68 \pm 1.78$ \\
\hline Serratia marcescens & $27.34 \pm 1.66$ & $25.29 \pm 1.43$ & $25.08 \pm 2.13$ \\
\hline Erwinia amylovora & $30.73 \pm 2.06$ & $27.12 \pm 1.83$ & $16.56 \pm 0.82$ \\
\hline Kluyvera cryocrescens & $28.77 \pm 4.67$ & $27.89 \pm 3.88$ & $19.64 \pm 1.13$ \\
\hline Yersinia enterocolitica & $22.74 \pm 0.95$ & 136 & \\
\hline
\end{tabular}

The cultivation time of enterobacteria on our modified Drigalski agar with lactose ranged from $16.28 \pm 1.44 \mathrm{~h}$ for Escherichia coli to $26.76 \pm 1.88 \mathrm{~h}$ for Shigella flexneri. Enterobacteria cultivation on Drigalski Lactose Agar (AppliChem) took from $19.64 \pm 1.13 \mathrm{~h}$ for Yersinia enterocolitica to $28.33 \pm 2.08 \mathrm{~h}$ for Shigella flexneri. At the same time, almost all enterobacterial strains isolated by us from wild animals formed colonies on modified Drigalski agar with lactose within $24 \mathrm{~h}$, with the exception of Kluyvera cryocrescens $(25.08 \pm 2.13 \mathrm{~h})$ and Shigella flexneri $(26.76 \pm 1.88 \mathrm{~h})$.

The cultivation time of enterobacteria isolated from zoo animals ranged from $17.06 \pm 3.78$ to $36.52 \pm 2.08 \mathrm{~h}$ (Table 5).

Table 5. Time of cultivation of enterobacteria isolated from zoo animals

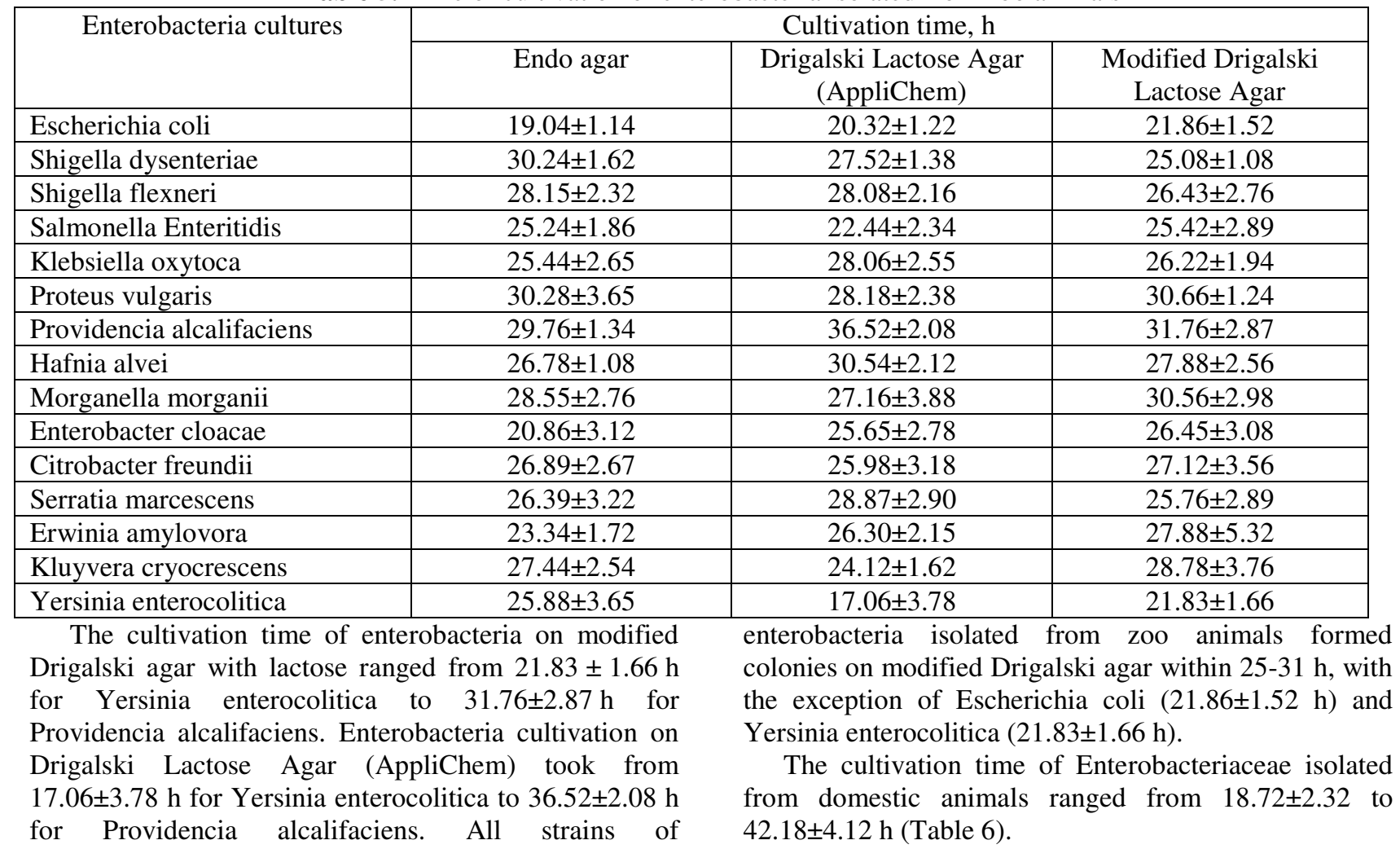


Table 6. Time of cultivation of enterobacteria, isolated from pets

\begin{tabular}{|l|c|c|c|}
\hline \multirow{2}{*}{ Enterobacteria cultures } & \multicolumn{3}{|c|}{ Cultivation time, $\mathrm{h}$} \\
\cline { 2 - 4 } & Endo agar & $\begin{array}{c}\text { Drigalski Lactose Agar } \\
\text { (AppliChem) }\end{array}$ & Modified Drigalski Lactose Agar \\
\hline Escherichia coli & $22.32 \pm 2.08$ & $26.46 \pm 2.58$ & $18.72 \pm 2.32$ \\
\hline Shigella dysenteriae & $38.66 \pm 2.74$ & $39.56 \pm 2.64$ & $33.45 \pm 2.45$ \\
\hline Shigella flexneri & $32.78 \pm 1.86$ & $36.44 \pm 2.82$ & $26.08 \pm 1.62$ \\
\hline Salmonella Enteritidis & $30.44 \pm 2.70$ & $26.86 \pm 1.52$ & $20.34 \pm 1.08$ \\
\hline Klebsiella oxytoca & $36.68 \pm 1.32$ & $30.18 \pm 1.14$ & $22.74 \pm 2.94$ \\
\hline Proteus vulgaris & $38.62 \pm 2.40$ & $26.14 \pm 2.56$ & $22.18 \pm 1.06$ \\
\hline Providencia alcalifaciens & $38.08 \pm 3.44$ & $35.74 \pm 3.84$ & $24.16 \pm 1.10$ \\
\hline Hafnia alvei & $30.04 \pm 2.64$ & $27.44 \pm 3.88$ & $25.90 \pm 3.70$ \\
\hline Morganella morganii & $29.89 \pm 3.18$ & $25.88 \pm 4.68$ & $23.80 \pm 1.68$ \\
\hline Enterobacter cloacae & $26.12 \pm 2.78$ & $28.70 \pm 1.44$ & $25.08 \pm 1.73$ \\
\hline Citrobacter freundii & $30.96 \pm 3.74$ & $28.52 \pm 3.56$ & $20.56 \pm 1.96$ \\
\hline Serratia marcescens & $33.94 \pm 2.18$ & $30.18 \pm 3.34$ & $24.62 \pm 2.06$ \\
\hline Erwinia amylovora & $38.08 \pm 3.64$ & $37.12 \pm 3.48$ & $26.50 \pm 2.46$ \\
\hline Kluyvera cryocrescens & $42.18 \pm 4.12$ & $38.68 \pm 3.08$ & $21.80 \pm 1.42$ \\
\hline Yersinia enterocolitica & $36.26 \pm 2.18$ & $30.48 \pm 2.82$ & \\
\hline
\end{tabular}

The cultivation time of enterobacteria on modified Drigalski agar with lactose ranged from $18.72 \pm 2.32 \mathrm{~h}$ for Escherichia coli to $33.45 \pm 2.45 \mathrm{~h}$ for Shigella dysenteriae. Cultivation of enterobacteria on Drigalski Lactose Agar (AppliChem) took from $25.88 \pm 4.68 \mathrm{~h}$ for Morganella morganii to $39.56 \pm 2.64 \mathrm{~h}$ for Shigella dysenteriae. Most enterobacterial strains isolated from domestic animals formed colonies on modified Drigalski agar within 18-27 h, with the exception of Shigella dysenteriae $(33.45 \pm 2.45 \mathrm{~h})$.

\section{Conclusion}

130 strains of enterobacteria were isolated from farm animals, with the total number of enterobacteria of $1.16^{\times} 10^{6} \pm 1.32$ per $1 \mathrm{~g}$ of feces. In wild animals, 35 strains of enterobacteria were isolated, with the total number of enterobacteria of $1.15^{\times} 10^{6} \pm 1.18$ in $1 \mathrm{~g}$ of feces. 23 strains of enterobacteria were isolated from zoo animals with the total number of enterobacteria of $1.59^{\times} 10^{6} \pm 1.46$ per $1 \mathrm{~g}$ of feces. 65 strains of enterobacteria were isolated from domestic animals, with the total number of enterobacteria of $1.53 \times 106 \pm$ 1.32 per $1 \mathrm{~g}$ of feces.

The species composition of enterobacteria isolated from various animal species consisted of the following representatives of the Enterobacteriaceae family: Escherichia, Shigella, Salmonella, Klebsiella, Proteus, Providencia, Hafnia, Morganella, Enterobacter, Citrobacter, Serratia, Erwinia, Kluyvera, Yersinia. The proportion of pathogenic enterobacteria in animals was not significant: Salmonella Enteritidis (0.012-0.24\%), Shigella dysenteriae and Shigella flexneri (0.003$0.01 \%)$, Klebsiella oxytoca $(2.44-3.12 \%)$, Yersinia enterocolitica $(0.04-0.12 \%)$. At the same time, shigella were not isolated from farm animals.

The ability to inactivate the factors of natural resistance of the macroorganism was more pronounced in pathogenic enterobacteria. Representatives of Salmonella Enteritidis, Shigella dysenteriae and
Shigella flexneri, Klebsiella oxytoca and Yersinia enterocolitica showed the highest indicators of antilysozyme and anticarnosine activity, the ability to biofilm formation.

The highest antimicrobial activity against all the enterobacteria we isolated was exhibited by carbenicillin from the group of carboxypenicillins and piperacillin from the group of ureidopenicillins, kanamycin, amikacin and gentamicin, cefepime from the group of cephalosporins of the IV generation, tetracycline, doxycycline, chloramphenicol, nalidixic acid, trimethoprim. Enterobacteria were highly resistant to benzylpenicillin from the group of natural penicillins, to streptomycin, cephalothin from the group of 1st generation cephalosporins, to polymyxin $\mathrm{B}$, to ofloxacin (tarivid) and metronidazole.

Microbiological dyes fuchsin basic, Congo red and water blue at a concentration of 0.05 to $50 \mathrm{~g} / \mathrm{dm}^{3}$, rosolic acid at a concentration of 0.05 to $1.0 \mathrm{~g} / \mathrm{dm}^{3} \mathrm{did}$ not affect the growth and reproduction of the enterobacteria we isolated. The dyes methyl red, methyl orange, bromcresol purple, phenol red, crystal violet, brilliant green, bromothymol blue, and gentian violet at a concentration of $0.1 \mathrm{~g} / \mathrm{dm}^{3}$ of the nutrient medium inhibited the growth and reproduction of enterobacteria. Andrade indicator at a concentration of $0.1 \mathrm{~g} / \mathrm{dm}^{3}$ had no effect on the growth and reproduction of enterobacteria. The BP indicator, consisting of aqueous blue and rosolic acid, at a concentration of 0.05 to $1 . \mathrm{g} / \mathrm{dm}^{3}$ did not affect the growth and reproduction of enterobacteria.

The formulation of the modified nutrient differential diagnostic medium Drigalski agar with lactose had the following composition (g/dm3): bacteriological agar - 12.0, pancreatic hydrolyzate of fishmeal - 5.0, pancreatic hydrolyzate of casein - 5.0, enzymatic bacteriological peptone with a high content of tryptophan - 5.0, aminopeptide 2.0, bread yeast extract -2.0 , gelatin -0.5 , sodium chloride -5.0 , sodium carbonate -0.5 , sodium sulfite -0.5 , sodium thiosulfate -0.3 , iron (II) sulfate -1.0 , basic fuchsine - 
1.0, Andrade indicator with BP indicator -0.2 . The medium was prepared according to recipe $\mathrm{A}$ and $\mathrm{B}$. Recipe A contained $10.0 \mathrm{~g} / \mathrm{dm}^{3}$ of lactose and $7.0 \mathrm{~g} / \mathrm{dm}^{3}$ of mannitol and recipe $\mathrm{B}$ contained 10.0 $\mathrm{g} / \mathrm{dm}^{3}$ of glucose and $7.0 \mathrm{~g} / \mathrm{dm}^{3}$ of sucrose.

The composition of the developed selective additive to modified Drigalski agar with lactose contained antibiotics vancomycin and telithromycin at a dose of $0.008 \mathrm{~g} / \mathrm{dm}^{3}, 0.004 \mathrm{~g} / \mathrm{dm}^{3}$ of linezolid.

The cultivation time of enterobacteria isolated from various animal species on the most commonly used differential diagnostic media ranged from 16.28 \pm 1.44 to $42.18 \pm 4.12 \mathrm{~h}$. Enterobacteria isolated from farm and wild animals formed colonies on modified Drigalski agar with lactose within $24 \mathrm{~h}$, while enterobacteria isolated from zoo animals formed colonies within 2531 hours. Enterobacteriaceae isolated from domestic animals formed colonies on modified agar within 18-27 hours. As a result, the modified Drigalski agar with lactose and a selective additive allowed reducing the cultivation time of enterobacteria isolated from various animal species.

\section{References}

1. B. Gelfand, B. Belotserkovsky, I. Milyukova, D. Protsenko, E. Gelfand, T. Popov, Annals of Surgery, 4, 12-26 (2015)

2. Yu. Kovtun, A. Kurilova, T. Taran, L. Katunina, N. Churikova, Problems of Particularly Dangerous Infections, 3, 92-95 (2014)

3. D. Dubrovskaya, R. Murzabaeva, A. Galieva, Infectious Diseases, 15 (51), 88 (2017)

4. N. Kozlova, Barantsevich N., Kosyakova K., Kameneva O., Barantsevich E., Bacteriology, 2 (3), 69-70 (2017)

5. A. Bykov, V. Zverev Medical Microbiology, Virology and Immunology (Atlas Guide, Moscow, 2018)

6. A.S. Labinskaya, Private medical microbiology with the technique of microbiological research (Textbook, Moscow, 2017)

7. A. S. Labinskaya, Guide to Medical Microbiology. Private medical microbiology and etiological diagnosis of infections. Book II (Textbook, Moscow, 2015)

8. A.S. Labinskaya, Guide to Medical Microbiology. Opportunistic infections: causative agents and etiological diagnosis. Book III. Volume I (Textbook, Moscow, 2013)

9. A.S. Labinskaya, Guide to Medical Microbiology. Clinical and epidemiological aspects. Book III. Volume II (Textbook, Moscow, 2014)

10. A. Shepelin, Bacteriology, 1, $42-47$ (2016)

11. A. Shelepin, Modern laboratory diagnostics, 2 (16), 18-20 (2015)

12. R.Yunusova, V. Gorelova, S. Kombarova, A. Aleshkin, O. Efimova, A. Bichucher, I. Martynenko, E. Alieva, Clinical laboratory diagnostics, 63 (2), 110-113 (2018)
13. R. Yunusova, V. Gorelova, S. Kombarova, A. Bichucher, I. Martynenko, E. Alieva, Laboratory Service, 5 (3), 63 (2016)

14. M.M. Medzhidov, Handbook on microbiological nutrient media (Handbook, Moscow, 2003)

15. D. Dubrovskaya, R. Murzabayeva, A. Mavzyutov, Bulletin of the Bashkir State Medical University, 3, 11-16 (2016)

16. N. Kozlova, N. Barantsevich, K. Kosyakova, O. Kameneva, S. Morozova, I. Churkina, Barantsevich E., Problems of Medical Mycology, 19 (1), 34-42 (2017)

17. N. Kozlova, N. Barantsevich, L. Ivanova, V. Goik, E. Barantsevich, Problems of Medical Mycology, 18 (3), 30-35 (2016) 